\title{
A Versatile Simulation-Assisted Layered Mesh Analysis for Generalized Litz Wire Performance
}

\author{
Noah J. Salk, Chathan M. Cooke \\ Laboratory for Electromagnetic and Electronic Systems, Massachusetts Institute of Technology, \\ Cambridge, MA 02139 USA
}

\begin{abstract}
This paper introduces a semi-analytical method of predicting AC loss in commercial Litz wires. Simple finite-element simulations are used to compute the resultant proximity fields in a coil system of arbitrary geometry. The approach addresses nonideal Litz wire construction by applying a surrogate skin effect model to inform and distribute the current over the cross-section into segmented layers. This simplifies the finite-element problem into a two-dimensional, DC simulation with a low number of mesh elements. Analytical solutions are then used to compute frequency-dependent loss due to skin and proximity effect. The method is demonstrated using two 14 AWG equivalent Litz wires with very different constructions and is validated with experimental results from several coil configurations. Finally, an appeal is made to commercial Litz wire manufacturers to provide an empirical "fabrication factor" specification that would allow consumers to predict the performance of a conductor in their application.
\end{abstract}

\section{NOMENCLATURE}

$\delta \quad$ Skin depth, $\sqrt{\frac{\rho}{\pi f \mu_{0}}}$

$\mu \quad$ Permeability of free space

$\rho \quad$ Conductor resistivity

$d_{s} \quad$ Strand diameter

$d_{\text {bos }} \quad$ Conductor diameter to the outer surface of the bundle

$F_{R} \quad$ Skin-effect resistance factor

$F_{f a b} \quad$ Fabrication factor

$G_{R} \quad$ Proximity loss factor

$I_{t o t} \quad$ Total peak current through the bundle

$N_{t} \quad$ Number of turns in the single-layer solenoid

$N_{s} \quad$ Total number of strands in the bundle

\section{INTRODUCTION}

The concept of using Litz wire to reduce AC associated losses was introduced nearly a century ago for radio applications [1]. The idea being that by breaking up a conductor into smaller insulated filaments or strands and using proper transposition, every strand in the conductor will see the same resistance over the lay. This means that the current is evenly distributed over the cross-section.

Litz wire began attracting more interest in recent years as wide-band gap (WBG) power semiconductors became an enabler for several high frequency, high power technologies, such as induction cooktops [2], EV wireless power transfer [3], megawatt-class electric machines [4], and resonant power transformers [5]. Many modeling approaches have been proposed for the optimization of Litz coil efficiency. Several analytical methods were established in the literature over the decades [6]-[11] all aimed at quick performance approximations. For more detailed predictions, three-dimensional numerical methods have been employed such as FEA [12] and the more novel partial element equivalent circuit (PEEC) method [13]-[15] which does not require the discretization of air. These 3D approaches are accurate given knowledge of the exact strand level construction, but suffer from computational limitations (memory, complexity, and long simulation times) due to the large number of mesh elements required.

There is an additional element of unknown in the actual construction of these Litz wires. A perfect Litz wire is technically possible to make, but the number of cabling operations required to do so renders these conductors economically impractical. Moreover, a DC resistance penalty is incurred with every twisting operation, resulting in a conductor with worse performance than an imperfect Litz wire with fewer fabrication levels. Actual fabrication techniques lead to randomized strand positions and inaccuracies in all of the aforementioned models due to non-idealities in the current distribution. Plumed attempted to address this by introducing some randomization in the strand placement of his simulations [12], but exact packing information is required to model reality. As such, most approaches in the literature use the ideal assumption that each strand in the bundle carries equal current. The key to improving Litz modeling accuracy is determining the non-ideal current distribution over the cross-section. If the current distribution is known, resultant fields can be computed and used to calculate losses as employed in other methods.

\section{LOSS MECHANISMS}

Nearly all analytical models established in the literature to compute AC loss in Litz wire attempt to take advantage of the exact solution for $\mathrm{AC}$ loss in two ideal scenarios: a straight cylindrical conductor carrying sinusoidal transport current and a straight cylindrical conductor placed in a sinusoidally varying homogenous and transverse magnetic field. These two cases are referred to as skin effect and proximity effect respectively, and the loss in each scenario is computed analytically in terms of Kelvin functions. Both loss mechanisms have been proven to be orthogonal [8] and therefore associated loss can be computed separately and summed up to calculate total AC loss.

A core premise to the calculation is if the AC current carried by every strand in the conductor and the resultant magnetic field amplitude local to every strand is known, then the loss in 
the Litz wire can be accurately computed with the assumption that the magnetic field applied to each strand is approximately homogeneous. These fundamental calculations are as follows.

\section{A. Skin Effect}

The current distribution within a straight, cylindrical conductor isolated in free space is known and is used to compute the $\mathrm{AC}$ resistance for any strand diameter and current injection frequency. Two ratio parameters are introduced here that will be used through-out the remainder of the paper and that collectively reduce the problem by two dimensions. The first parameter, $F_{R}$, is the ratio of the $\mathrm{AC}$ resistance due to skin effect to the DC resistance of the conductor [7]. The second parameter, $\frac{d_{s}}{2 \delta}$, is the ratio of the strand diameter to two times the skin-depth. The exact skin effect resistance ratio of the single conductor case is well known [16] and can be expressed in terms of Kelvin functions (definitions of which can be found in the Appendix) using a slight variation of $\frac{d_{s}}{2 \delta}, \gamma_{s}=\frac{d_{s}}{\sqrt{2} \delta}$ to simplify the notation,

$$
F_{R}=\frac{\gamma_{s}}{2} \frac{\operatorname{ber}\left(\gamma_{s}\right) b e i^{\prime}\left(\gamma_{s}\right)-\operatorname{bei}\left(\gamma_{s}\right) b e r^{\prime}\left(\gamma_{s}\right)}{b e r^{\prime 2}\left(\gamma_{s}\right)+b e i^{\prime 2}\left(\gamma_{s}\right)}
$$

For a cylindrical strand carrying sinusoidal AC current with amplitude $I_{\text {peak }}$, the power loss per-unit-length due to the skin effect is,

$$
P_{\text {skin }}=R_{D C} F_{R} \frac{1}{2} I_{\text {peak }}^{2}=\frac{4 \rho}{\pi d_{s}^{2}} F_{R} \frac{1}{2} I_{\text {peak }}^{2}
$$

where $\rho$ is the DC resistivity of the conductor material.

\section{B. Proximity Effect}

Likewise, for a straight cylindrical conductor present in a homogeneous transverse AC magnetic field, there exists an exact solution to the power loss per-unit-length due to the proximity effect. Similar to $F_{R}$, the proximity factor, $G_{R}$, is dependent only on the strand geometry, resistivity $(\rho)$, and operating frequency (via its effect on the skin depth, $\delta$ ). It is defined as [8],[17],

$$
G_{R}=2 \pi \gamma_{s} \rho \frac{\operatorname{ber}_{2}\left(\gamma_{s}\right) b e r^{\prime}\left(\gamma_{s}\right)+b e i_{2}\left(\gamma_{s}\right) b e i^{\prime}\left(\gamma_{s}\right)}{\operatorname{ber}^{2}\left(\gamma_{s}\right)+b e i^{2}\left(\gamma_{s}\right)}
$$

The calculation for proximity loss per-unit-length is written in terms of only the proximity factor and the peak amplitude of the applied AC magnetic field, $H_{\text {peak }}$,

$$
P_{\text {prox }}=G_{R} H_{\text {peak }}^{2}
$$

The total loss is the numerical sum of the skin- and proximity effect losses.

$$
P_{\text {loss }}=P_{\text {skin }}+P_{\text {prox }}
$$

\section{AdDRESSING NON-IDEAL CONSTRUCTION}

If the conductor of interest is perfectly "Litzed" (every strand spends equal time in every position within the crosssection) over the length of the wire, then each strand carries equal current and the current density in the conductor's cross section is approximately uniform. This case results in several simplifications and is the basic approximation for the majority of analytical methods in the literature [7]-[11]. However, manufacturing a perfect Litz wire requires many cabling operations that not only add complexity/cost, but also increase the DC resistance of the conductor. Therefore, a fabrication shortcut called bunching is used in which the base level is composed of many essentially parallel strands that are then packed and twisted together. This operation does not result in full radial transposition for every strand and therefore renders the assumption of uniform current density throughout the cross-section invalid.

\section{A. Informed Geometry Generation}

Litz wire is a multi-level structure consisting of two types of operations: cabling (denoted by ' $\mathrm{x}$ ' or '( )' for served cabling) and bunching (denoted by ' $/$ '). Cabling typically happens at the higher levels and is the process of twisting two or more concentric sub-bundles together. To ensure structural integrity and proper azimuthal transposition, the number of sub-bundles cabled at any given level is often limited to about 7 but is sometimes increased at the highest level. The bunched operation is when a given number of strands are collectively twisted without enforcement of full radial transposition. A perfectly "Litzed" wire is constructed using only cabling operations.

As an example, the manufacturing process for a $5 \times 5 \times 42 / 44$ AWG Litz wire in chronological order (lowest to highest level) is: 42 strands of 44 AWG magnet wire are gathered together and twisted, 5 of these bunched bundles of 42 strands are cabled together, and finally 5 of those resulting sub-bundles are cabled together to create a conductor with 1050 total strands. The levels of construction can be seen in the side section view of this conductor in Fig. 1(a). As the conductor was initially served with Nomex insulation, Fig. 1(b) shows a highly packed

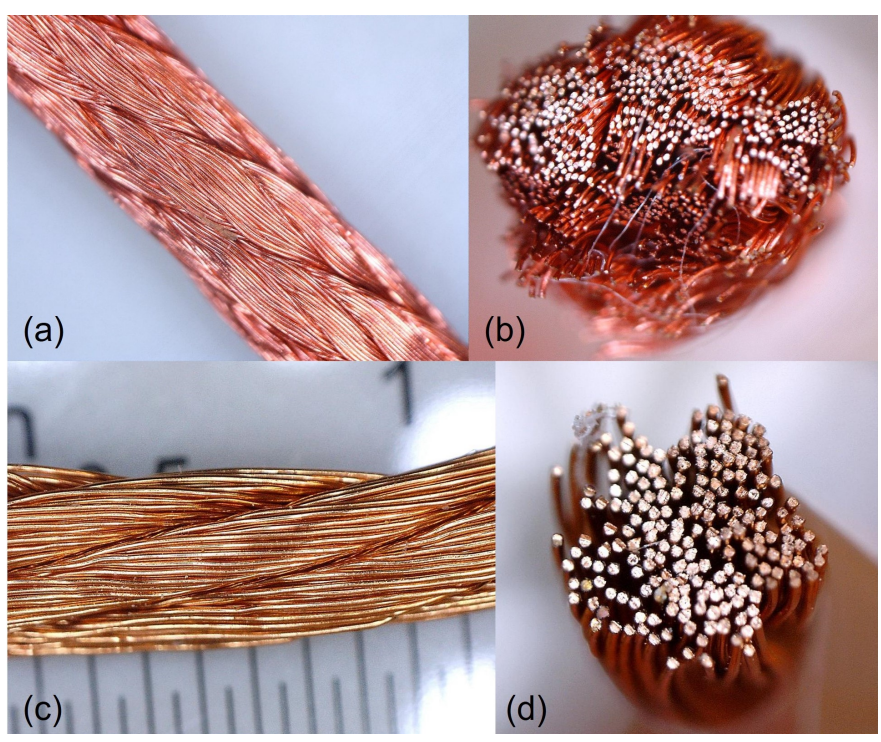

Fig. 1. Close-ups of both the $5 \times 5 \times 42 / 44$ AWG Litz wire's (a) side view, and (b) cross-section and 5x40/36 AWG Litz wire's (c) side view, and (d) cross-section. Pictures captured after removing the Nomex serving. 
cross-section. Manufacturers typically twist successive cabling operations in opposite directions.

The guiding principle of the proposed loss calculation method is to model the non-idealities resulting from the bunching operation and thereby enable unequal current sharing between strands from the ideal assumption of equal distribution, hence affecting the field distribution within the crosssection. All cabling operations are assumed to be perfect, such that each bunched sub-bundle carries equal total current. Because the bunched level is twisted together, each strand at the perimeter should carry the same current. Likewise, each strand in subsequent layers inside the bunched sub-bundle should carry the same current as the others in their layer. This is modeled by creating "concentric" layers of thickness equal to a strand diameter as shown in Fig. 2(a). The current density within a layer is approximated as uniform and is to be determined in the following sections.

The sub-bundles are fit into a circular cross-section to simulate a tightly packed conductor. Each packed shape, shown as "triangular" and "rectangular" sectors in Fig. 2(a) should have equal area, but the exact geometry is arbitrary. This is because the actual shape after packing is not defined; this variation will be encapsulated in the constant fabrication factor.

The 5x5x42/44 AWG Litz wire is a three level construction. The complexity of the packed geometry is more severe with more levels. A two level 5x40/36 AWG Litz wire example is shown in Fig. 1(c-d) and is much simpler to model. The layered mesh packed geometry for this conductor is shown in Fig. 2(b).

\section{FEA Simulation Assistance}

\section{A. Surrogate Skin Effect Model}

A surrogate cylindrical conductor skin effect model is used to inform and distribute the current in each layer of the layered-model Litz wire cross-section. The surrogate conductor is cylindrical and has a diameter equal to $2 m d_{s}$ where $m$ is the number of layers in the bunched level cross-section of interest. Again, the layers have thickness equal to the strand diameter, $d_{s}$, as shown in Fig. 3.

The current density in each layer is spatially averaged at the excitation frequency of interest as shown. An example of the quantized current density as a function of radius in the surrogate model is shown in Fig. 4. This can be computed from FEA or calculated analytically using the exact solution for the current distribution in an isolated cylindrical conductor carrying sinusoidal transport current. The total current through the surrogate conductor is unimportant since the resulting average current densities will be normalized and used relatively. Therefore, we will set the total peak current through the surrogate model as $1 \mathrm{~A}$ to simplify the math. The average current density in the $i^{\text {th }}$ layer is computed as,

$$
J_{i}=\frac{k}{\pi m d_{s}^{3}(2 i-1)} \int_{(i-1) d_{s}}^{i d_{s}} \frac{\mathcal{J}_{0}(k r)}{\mathcal{J}_{1}\left(k m d_{s}\right)} r d r
$$

where $\mathcal{J}_{0}$ and $\mathcal{J}_{1}$ are the Bessel functions of the first kind, orders zero and one respectively, and the wave number, $k$, is

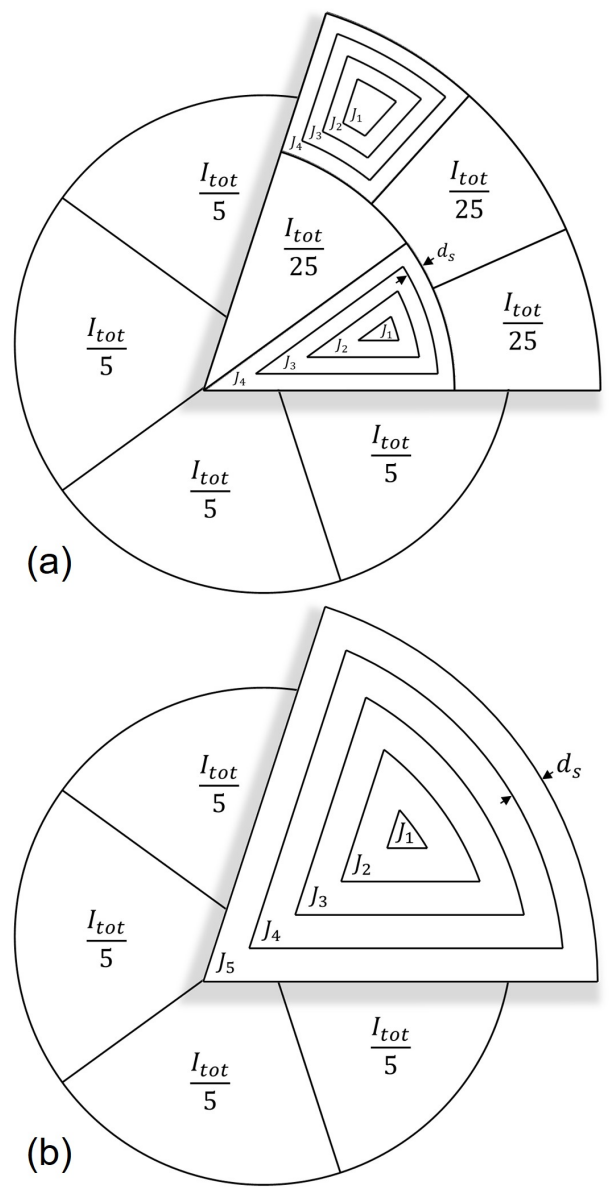

Fig. 2. Packed layer geometry used for the (a) $5 \times 5 \times 42 / 44$ AWG and (b) $5 \times 40 / 36$ AWG Litz wire analysis; expanded sub-bundles are shown carrying $I_{\text {tot }} / 5$

defined as $\frac{1-j}{\delta}$. From this average current density, the current in each layer of the Litz wire is computed and scaled to attain the total injection current peak amplitude, $I_{t o t}$,

$$
I_{i}=\frac{I_{t o t} J_{i} A_{i}}{\beta \sum_{n=1}^{m} J_{n} A_{n}}
$$

where $\beta$ is the fraction of the total current carried at the bunched level. This number would be 25 for the $5 \times 5 \times 42 / 44$ AWG Litz wire and 5 for the $5 \times 40 / 36$ AWG Litz wire. Additionally, $A_{i}$ is the area of the $i^{\text {th }}$ layer in the Litz wire cross-section. This area changes depending on the geometry of the bunched-level cross-section and must be computed individually for the triangular and rectangular sectors.

\section{B. Loss Calculation}

The current distribution has already been determined using the surrogate skin effect model. It is therefore possible to calculate skin effect loss in the conductor before simulation. Generally, this is computed from (2) as follows,

$$
P_{\text {skin }}=\frac{4 \rho F_{R}}{\pi d_{s}^{2}} \sum_{i}^{\Omega} \frac{\pi d_{\text {bos }}^{2}}{4 N_{s} A_{i}} \frac{1}{2} I_{i}^{2}
$$




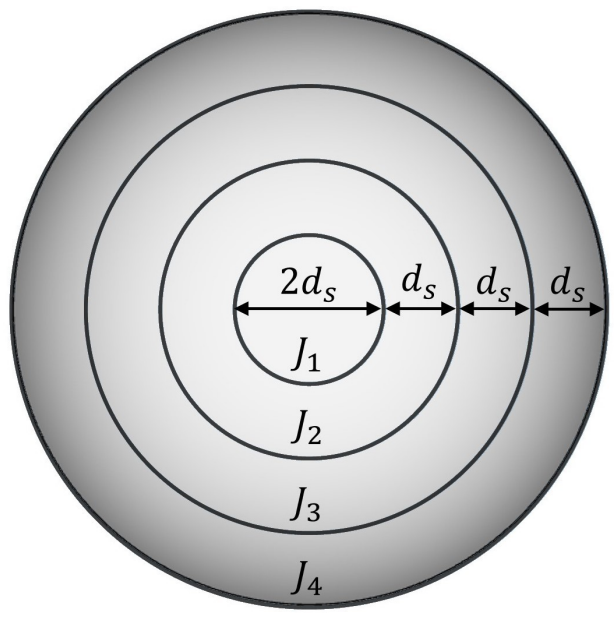

Fig. 3. Surrogate skin effect model for layered current distribution, $m=4$

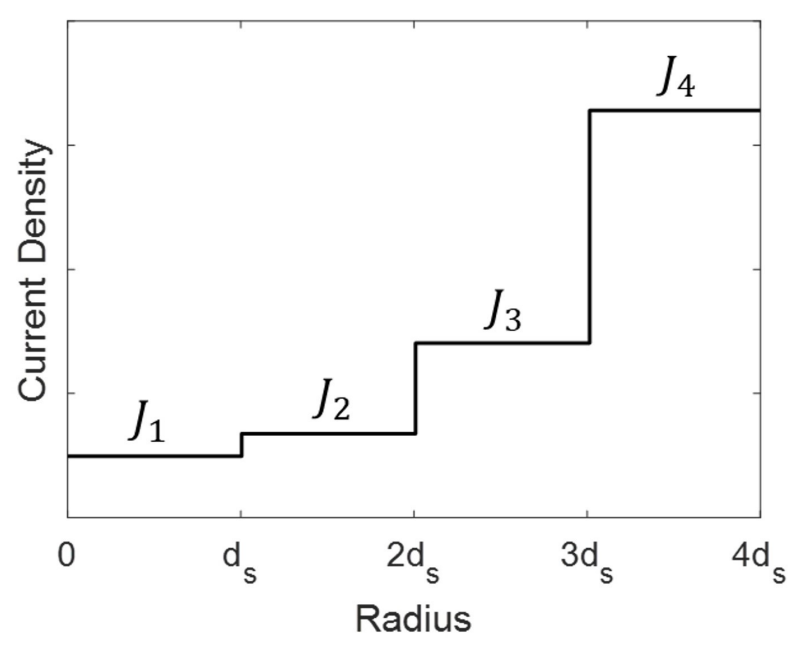

Fig. 4. Example of the quantized current density in each layer, $m=4$

where $\Omega$ is the conductor cross-section domain, $N_{s}$ is the total number of strands in the bundle, and $d_{b o s}$ is the conductor diameter to the outer surface of the bundle. This is essentially computing the number of strands in each layer as the product of the total number of strands in the conductor and the fraction of the cross-sectional area covered by each layer. For each layer, the number of strands and the current carried, $I_{i}$ (assumed to be distributed equally amongst each strand), is enough information to compute the power loss due to skin effect for that layer. The total skin effect loss is the summation of this for each layer in the bundle.

The $5 \times 5 \times 42 / 44$ AWG conductor is used here as an example to demonstrate the full skin effect power loss computation. If we define $A_{t, i}$ and $A_{r, j}$ as the area of the $i^{\text {th }}$ layer in the triangular sector and $j^{\text {th }}$ layer in the rectangular sector respectively, the total area of the conductor's cross section is,

$$
A_{\Omega}=10 \sum_{i=1}^{4} A_{t, i}+15 \sum_{j=1}^{4} A_{r, j}=\frac{\pi d_{b o s}^{2}}{4}
$$

The total number of strands that belong to the $i^{\text {th }}$ layer of all of the triangular sectors in the bundle is,

$$
n_{t, i}=\frac{10 A_{t, i}}{A_{\omega}} N_{s}=\frac{40 A_{t, i}}{\pi d_{b o s}^{2}} N_{s}
$$

where $n_{t, i}$ is allowed to be a fraction. The current per strand in that layer is,

$$
\begin{aligned}
i_{t, i}=\frac{10 I_{t, i}}{n_{t, i}}=\frac{10 I_{t o t} J_{i} A_{t, i}}{25 n_{t, i} \sum_{n=1}^{4} J_{n} A_{t, n}} \\
\Rightarrow \frac{\pi d_{b o s}^{2} I_{t o t} J_{i}}{100 N_{s} \sum_{n=1}^{4} J_{n} A_{t, n}}
\end{aligned}
$$

Similarly, the total number of strands that belong to the $j^{\text {th }}$ layer of all of the rectangular sectors in the bundle is,

$$
n_{r, j}=\frac{15 A_{r, j}}{A_{\omega}} N_{s}=\frac{60 A_{r, j}}{\pi d_{b o s}^{2}} N_{s}
$$

and the current per strand in that layer is,

$$
\begin{aligned}
i_{r, j}=\frac{15 I_{r, j}}{n_{r, j}}=\frac{15 I_{\text {tot }} J_{j} A_{r, j}}{25 n_{r, j} \sum_{n=1}^{4} J_{n} A_{r, n}} \\
\Rightarrow \frac{\pi d_{\text {bos }}^{2} I_{\text {tot }} J_{j}}{100 N_{s} \sum_{n=1}^{4} J_{n} A_{r, n}}
\end{aligned}
$$

Finally, the total power loss per-unit-length due to skin effect is computed as,

$$
P_{\text {skin }}=\frac{2 \rho F_{R}}{\pi d_{s}^{2}}\left(\sum_{i=1}^{4} n_{t, i} i_{t, i}^{2}+\sum_{j=1}^{4} n_{r, j} i_{r, j}^{2}\right)
$$

The average peak magnetic field squared in the cross-section of the bundle is needed to calculate proximity loss. The purpose of using a finite-element simulation is to compute the magnetic field within the cross-section that results from the current distribution assigned in the previous section. A twodimensional, DC simulation is setup to capture the current assignments for each layer since the frequency dependence at the bundle level has already been taken care of using the surrogate model. It is important to note that the current must be redistributed at every frequency according to the results of the surrogate model.

The spatially averaged resultant magnetic field amplitude squared is computed over the conductor cross-section from simulation results,

$$
\left\langle H_{\text {peak }}^{2}\right\rangle=\frac{1}{A_{\Omega}} \int_{\Omega}\left|H_{\text {peak }}\right|^{2} d A
$$

In the isolated conductor case, $A_{\Omega}=\frac{\pi d_{b o s}^{2}}{4}$, but in a single coil it would be $A_{\Omega}=\frac{N_{t} \pi d_{b o s}^{2}}{4}$, where $N_{t}$ is the number of turns in the coil. This makes calculating proximity loss perunit-length (which is proportional to $H_{p e a k}^{2}$ ) easier by taking the average field amplitude squared as the local transverse magnetic field applied to each strand in the conductor and plugging this into (4),

$$
P_{\text {prox }}=N_{s} G_{R}\left\langle H_{\text {peak }}^{2}\right\rangle
$$



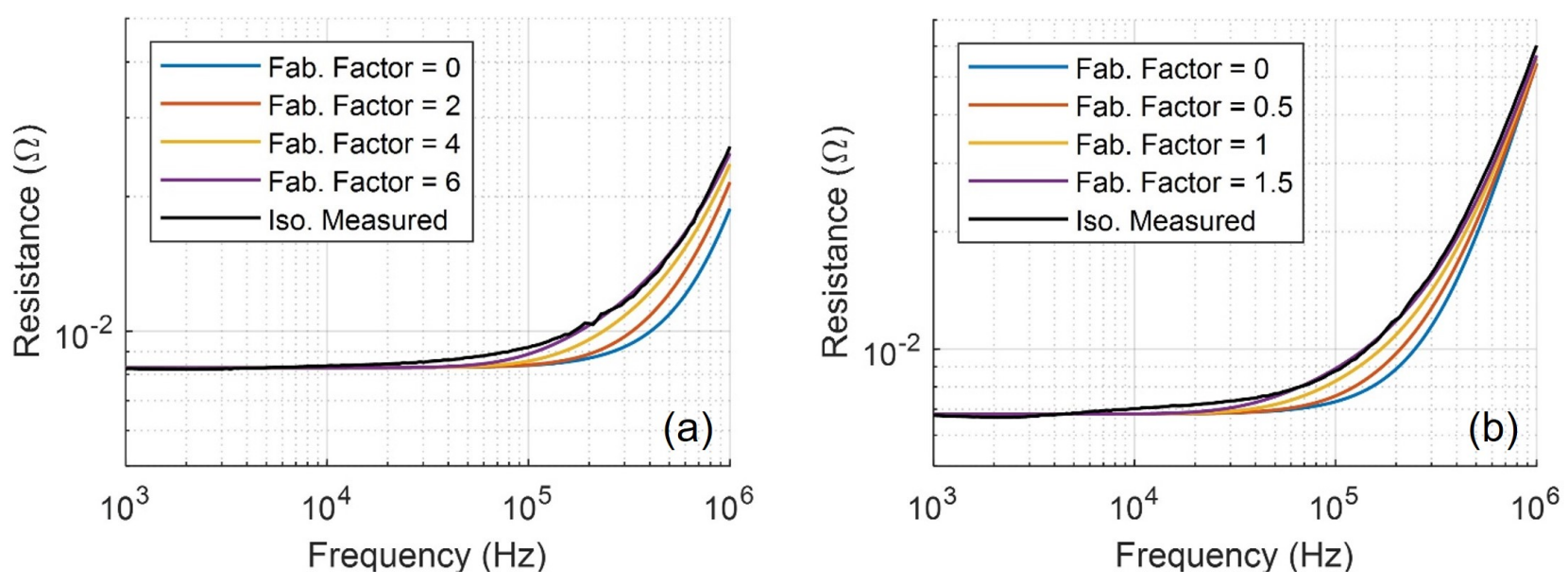

Fig. 5. Isolated conductor layered mesh analysis with varying fabrication factors on the (a) $5 \times 5 \times 42 / 44$ AWG Litz wire $(m=4)$ and (b) $5 \times 40 / 36$ AWG Litz wire $(m=5)$ compared to their respective isolated conductor (zig-zag) measurements

The per-unit-length AC resistance is then computed from the summation of the skin effect loss and proximity effect loss in the conductor,

$$
R_{A C}=\frac{2}{I_{\text {tot }}^{2}}\left(P_{\text {skin }}+P_{\text {prox }}\right)
$$

\section{Fabrication Factor Extraction}

There are two factors that make the surrogate skin effect model not immediately applicable to the layered mesh geometry:

1) The triangular and rectangular sectors used in the crosssection geometry does not exactly depict reality; the actual shapes are random and impossible to predict with accuracy

2) The bunching operation results in some radial transposition and current sharing between layers

To address these unknowns, an empirical fabrication factor is used. This fabrication factor is unique to each Litz conductor construction, but is independent of the application of that Litz wire; therefore, it is a single value which can be supplied by the manufacturer as determined from a standardized isolated conductor test, for example the "zig-zag" test as discussed below. The fabrication factor is extracted from measurements obtained with an isolated conductor scenario. The measurement here is made using a "zig-zag" flat board pattern (shown in Fig. 6) in which an even number of loops are made to

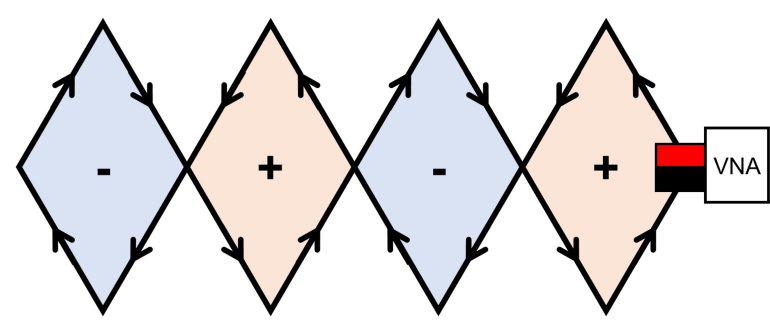

Fig. 6. Zig-zag isolated conductor measurement setup

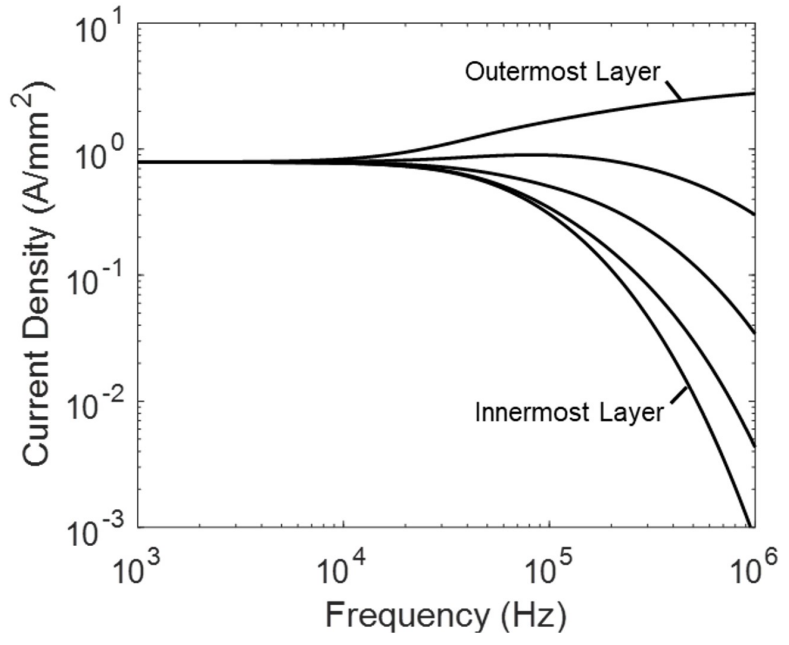

Fig. 7. Current density in each layer of the $5 x 40 / 36$ AWG Litz conductor with $F_{f a b}=1.5$ and excitation of $1 \mathrm{~A}$ as a function of frequency; extracted from the surrogate model

cancel out external fields. The conductor is always sufficiently far apart from itself such that the magnetic field generated by current across the board is not strong enough to induce additional proximity loss. The difference between the isolated conductor measurements and analytical theory [8] is due to non-idealities in the construction that results in unequal current distribution throughout the cross-section, which are better represented in the new calculation presented here.

The fabrication factor, $F_{f a b}$, is a multiplicative factor applied to every frequency of interest prior to running the surrogate skin effect model. Doing this scales the severity of the bundle-level skin effect. The skin-depth, $\delta$, used in calculating the wave number for the surrogate model in (6) is then replaced by $\delta_{e f f}$,

$$
\delta=\sqrt{\frac{\rho}{\pi f \mu}} \Rightarrow \delta_{\text {eff }}=\sqrt{\frac{\rho}{\pi F_{f a b} f \mu}}
$$



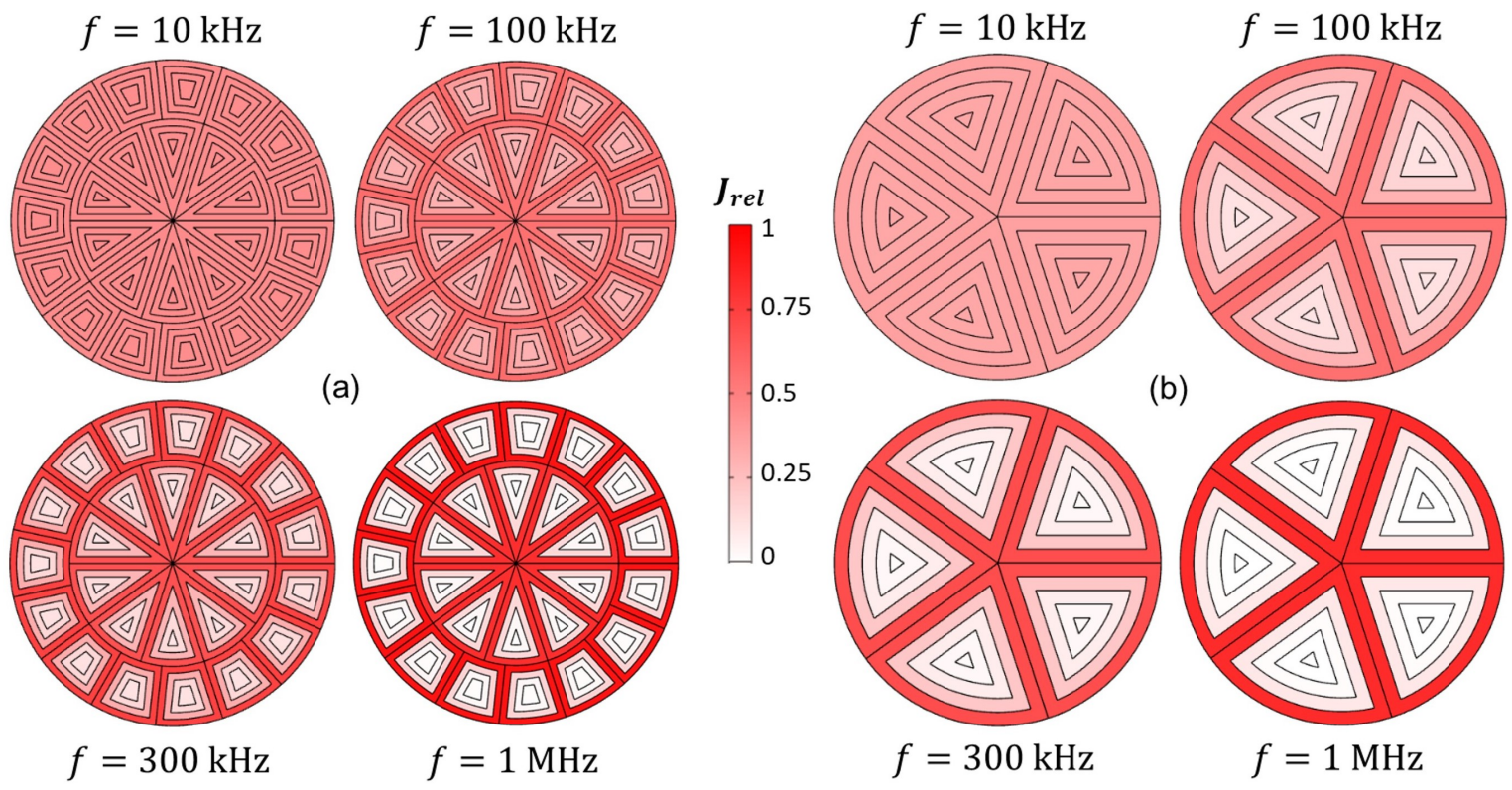

Fig. 8. Individually normalized current density distributions in each layer at 4 different frequencies for the (a) $5 \times 5 \times 42 / 44$ AWG conductor $\left(F_{f a b}=6\right)$ and (b) $5 \times 40 / 36$ AWG conductor $\left(F_{f a b}=1.5\right)$

where $\mu$ is the magnetic permeability of the conductor.

In order to determine the fabrication factor, the layered mesh analysis is run under the isolated conductor case with varying frequency multipliers. Once the frequency dependent resistance of the isolated layered mesh analysis matches "zigzag" isolated measurements, the frequency multiplier is taken as the fabrication factor. This process is shown in Fig. 5 for both the 5x5x42/44 AWG and 5x40/36 AWG Litz wires. Note that a fabrication factor of zero is equivalent to the uniform cross-sectional current density assumption used by analytical models in the literature.

From two plots in Fig. 5, a fabrication factor of 6 for the $5 \times 5 \times 42 / 44$ AWG Litz wire matches the measured results very well, whereas for the $5 \times 40 / 36$ AWG Litz wire, a fabrication factor of 1.5 matches the measured results. These two values are used as the fabrication factor values for future analysis on each respective conductor when applied to any coil or other configuration. The current density distribution between layers in the 5x40/36 AWG Litz wire, as determined by the surrogate method, is given in Fig. 7 as a function of frequency and using the fabrication factor of 1.5. Currents in each layer of the conductor cross-section are set prior to simulation as shown in Fig. 8 for both conductors at 4 different frequencies. The non-uniform current distribution of the layered mesh method results in more accurate internal field representation and hence improved associated loss calculations.

\section{Arbitrary Coil Geometry}

The twisting at all levels of the Litz wire construction has the effect of canceling out flux linkages induced due to transverse external magnetic fields. This means that while the external fields cannot be ignored when calculating strand-level proximity loss, they do not change the current distribution in the cross section. This is why the fabrication factor is independent of application and makes simulations of arbitrary coil geometries straight forward.

In the axisymmetric solenoid simulation, the resultant magnetic field squared is averaged over all turns in the coil and then used to compute the aggregate proximity loss per-unitlength by applying this averaged field squared to each strand in the conductor.

\section{SOLENOID EXPERIMENTAL RESUlts}

The two Litz conductors were each wound onto respective 6 inch OD PMMA cylinders in 6 and 12 turn coil configurations using 3D printed clips to set desired turn-to-turn spacing. Fig. 9 shows the $5 \times 5 \times 42 / 44$ AWG served conductor wound into 12 turns with c/a spacing factors of 1.5 and 2.5 respectively. Note that the spacing factor is the coil pitch, $\lambda$, normalized by the conductor OD, $c / a=\lambda / d_{\text {bos }}$.

Measurements were taken via an Omicron Bode 100 VNA with the one-port method (see Appendix for more details). These measured results are compared to the layered mesh

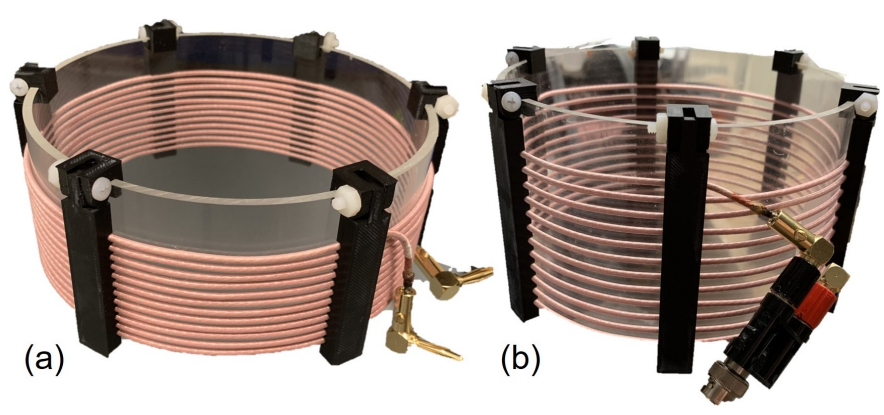

Fig. 9. 12 turn 5x5x42/44 AWG Litz coils with (a) $c / a=1.5$ (b) $c / a=2.5$ 

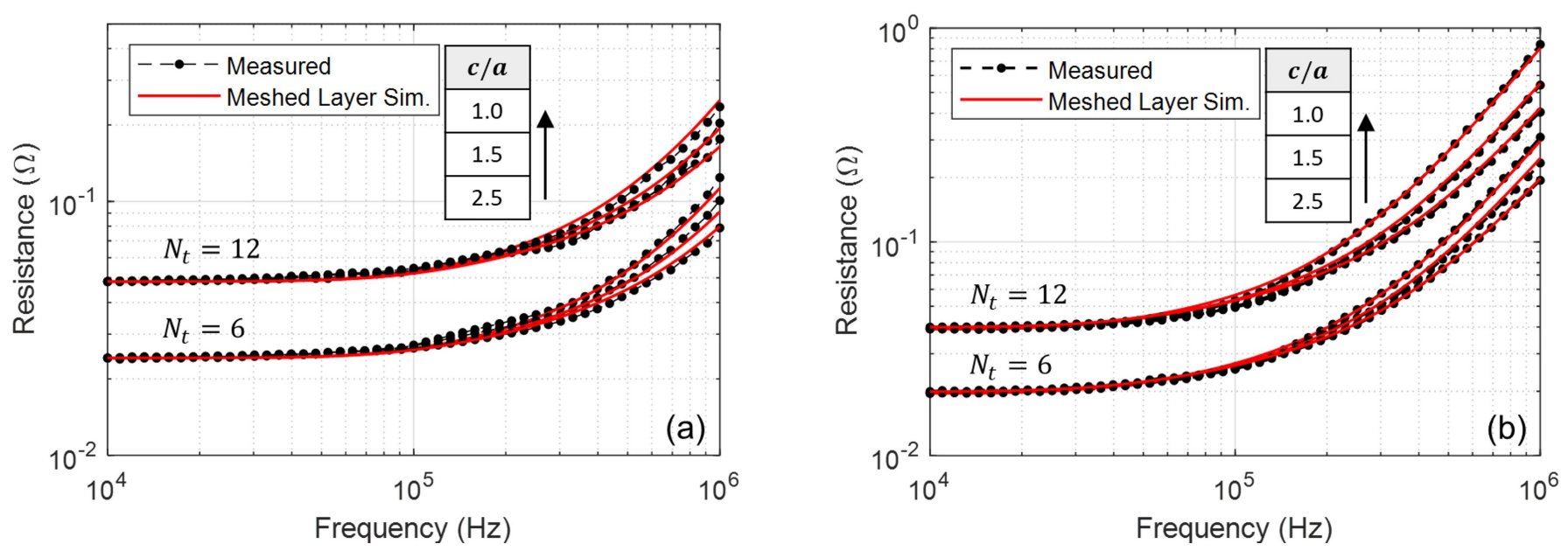

Fig. 10. Simulation and measurement results for several coil configuration using the (a) $5 \times 5 \times 42 / 44$ AWG Litz wire and (b) 5 AWG Litz wire and (b) $5 \times 40 / 36$ AWG Litz wire

simulation in Fig. 10. In order to accurately assess the performance of the simulation method, the measurements were linearly scaled to match the simulated resistance value at 10 $\mathrm{kHz}$. This absolute deviation from theory in measured DC resistance (up to 15\%) can be attributed to several different factors, including lead connections, imperfect termination (soldered), and limited ability of the measurement method at low impedances. The linear scaling correction was proven to be the proper compensation approach by applying the same scaling to measurements of solid conductor coils and comparing to FEA simulated results (see Appendix). All FEA simulations were carried out in COMSOL's AC/DC module.

\section{CONCLUSION}

The layered mesh simulation method of Litz conductor loss calculations provides a fast alternative to more computationally intensive three-dimensional simulation methods and accounts for non-idealities in the bundle construction. The method is general enough that it may be applied to arbitrary conductor applications for a given packed layer geometric representation of a Litz bundle. A unique corresponding fabrication factor used in the loss calculation for a given Litz wire is defined, and is a quantity that could be supplied by a manufacturer as obtained from isolated conductor tests.

The calculated resistance from the proposed loss approximation method closely matched experimental results obtained from several different coil configurations and with two Litz wires of equivalent gauge but very different construction. The authors suggest the use of a purely analytical method of loss calculation such as [11] for design space exploration and then, due to the initial complexity of setting up the cross-sectional geometry for a given conductor (though this may be solved through a provided drawing file i.e. dxf), using the proposed method for finer detailed analysis when choosing a conductor arrangement from a narrowed down set of choices. Future work will focus on adopting the layered mesh simulation method to multi-coil system analysis.

\section{APPENDIX}

\section{A. Mathematical Definitions}

Definitions for the Kelvin functions used in (1) and (3) are written below, where $\mathcal{J}_{n}(\theta)$ is the $n^{\text {th }}$ order Bessel function of the first kind [18].

$$
\begin{gathered}
\operatorname{ber}(x)=\operatorname{ber}_{0}(x) \\
\operatorname{bei}(x)=\operatorname{bei}_{0}(x) \\
\operatorname{ber}_{n}(x)=\operatorname{Re}\left\{\mathcal{J}_{n}\left(x e^{\frac{j 3 \pi}{4}}\right)\right\} \\
\operatorname{bei}_{n}(x)=\operatorname{Im}\left\{\mathcal{J}_{n}\left(x e^{\frac{j 3 \pi}{4}}\right)\right\} \\
\operatorname{ber}^{\prime}(x)=\frac{\operatorname{ber}_{1}(x)+b e i_{1}(x)}{\sqrt{2}} \\
\operatorname{bei}^{\prime}(x)=\frac{\operatorname{bei}_{1}(x)-b e r_{1}(x)}{\sqrt{2}}
\end{gathered}
$$

\section{B. Solid Conductor Coil as a Calibration Gauge}

Taking low resistance measurements at high frequencies is challenging for any impedance analyzer or vector network analyzer (VNA), especially for coils with significant inductance. The reactance of a coil at high frequencies can be several orders of magnitude higher than the resistance component. This means the measurement equipment must be sensitive enough to detect very small phase differences.

The Omicron Bode 100 VNA was used for all of the measurements taken in this paper. Several measurement methods are possible with a VNA and Omicron's manual provides the suggested method range depending upon the frequency sweep of measurement and impedance magnitude of the device under test (DUT). The "one-port" method is recommended for the coils measured in this paper and was shown via a sensitivity study to be the best method. To verify the calibration quality, the open, load, and short calibrations were run with care and several coil configurations using a 14 AWG solid magnet wire 
were then measured. Turn-to-turn spacing was fixed at three different set values using 3D printed coil forming clips. The solid conductor coil geometry is well defined and therefore measurements results should match simple 2D axisymmetric FEA simulation results. The coil measurements were found to closely follow FEA after linearly scaling to match DC resistances. The authors suggest gauging calibration quality with a fixed coil of known frequency dependent resistance prior to the measurement of the more complex Litz wire coils.

\section{ACKNOWLEDGEMENT}

This work was supported by ProlecGE and the first author was supported in part by the EE Landsman Fellowship fund at the Massachusetts Institute of Technology. The authors would like to thank Carlos Gaytan Cavazos of ProlecGE for his technical advise.

\section{REFERENCES}

[1] S. Butterworth, "Effective resistance of inductance coils at radio frequency," Wireless and Wireless Eng., vol. 3, p. 483, 1926.

[2] J. Acero, J. Burdio, L. Barragan, D. Puyal, and R. Alonso, "Frequencydependent resistance in litz-wire planar windings for all-metal domestic induction heating appliances," Twentieth Annual IEEE Applied Power Electronics Conference and Exposition, 2005. APEC 2005.

[3] A. Kurs, A. Karalis, R. Moffatt, J. D. Joannopoulos, P. Fisher, and M. Soljacic, "Wireless power transfer via strongly coupled magnetic resonances," Science, vol. 317, no. 5834, p. 83-86, 2007.

[4] A. Yoon, X. Yi, J. Martin, Y. Chen, and K. Haran, "A high-speed, highfrequency, air-core pm machine for aircraft application," 2016 IEEE Power and Energy Conference at Illinois (PECI), 2016.

[5] A. J. Carvajal, "First principles design of coreless power transformers," Master's thesis, Massachusetts Institute of Technology, Cambridge, MA, 2016.

[6] F. E. Terman, "Radio engineering handbook," Journal of the British Institution of Radio Engineers, vol. 11, no. 1, p. 35-35, 1951.

[7] P. Dowell, "Effects of eddy currents in transformer windings," Proceedings of the Institution of Electrical Engineers, vol. 113, no. 8, p. 1387, 1966.

[8] J. Ferreira, "Improved analytical modeling of conductive losses in magnetic components," IEEE Transactions on Power Electronics, vol. 9, no. 1 , p. 127-131, 1994.

[9] F. Tourkhani and P. Viarouge, "Accurate analytical model of winding losses in round litz wire windings," IEEE Transactions on Magnetics, vol. 37, no. 1, p. 538-543, 2001.

[10] M. Bartoli, N. Noferi, A. Reatti, and M. Kazimierczuk, "Modeling litzwire winding losses in high-frequency power inductors," PESC Record. 27th Annual IEEE Power Electronics Specialists Conference, 1996.

[11] X. Nan and C. Sullivan, "An improved calculation of proximity-effect loss in high-frequency windings of round conductors," IEEE 34th Annual Conference on Power Electronics Specialist, 2003. PESC 03.

[12] E. Plumed, I. Lope, C. Carretero, and J. Acero, "A recursive methodology for modelling multi-stranded wires with multilevel helix structure," Applied Mathematical Modelling, vol. 83, p. 76-89, 2020.

[13] R. Y. Zhang, J. K. White, J. G. Kassakian, and C. R. Sullivan, "Realistic litz wire characterization using fast numerical simulations," 2014 IEEE Applied Power Electronics Conference and Exposition - APEC 2014, 2014.

[14] S. Ehrlich, H. Rossmanith, M. Sauer, C. Joffe, and M. Marz, "Fast numerical power loss calculation for high-frequency litz wires," IEEE Transactions on Power Electronics, vol. 36, no. 2, p. 2018-2032, 2021.

[15] J. Lyu, H. Chen, Y. Zhang, Y. Du, and Q. S. Cheng, "Fast simulation of litz wire using multilevel peec method," IEEE Transactions on Power Electronics, vol. 35, no. 12, p. 12612-12616, 2020.

[16] G. S. Smith, "A simple derivation for the skin effect in a round wire," European Journal of Physics, vol. 35, no. 2, p. 025002, 2014.

[17] M. Perry and T. Jones, "Eddy current induction in a solid conducting cylinder with a transverse magnetic field," IEEE Transactions on Magnetics, vol. 14, no. 4, p. 227-232, 1978 .
[18] J. W. W., A. Young, and A. Kirk, "Bessel functions. part iv, kelvin functions," Mathematics of Computation, vol. 19, no. 90, p. 344, 1965. 